\title{
Medium effects on subthreshold kaon production in heavy-ion collisions
}

\author{
X. S. Fang, C. M. Ko, G. Q. Li, and Y. M. Zheng* \\ Cyclotron Institute and Physics Department, Texas A $8 M$ University, College Station, Texas 77843
}

(Received 16 August 1993; revised manuscript received 4 November 1993)

\begin{abstract}
The relativistic transport model is extended to include the kaon degree of freedom. We also take into account the density dependence of the kaon effective mass in nuclear matter and the rescattering of kaons by nucleons. We find that the inclusion of kaon self-energy due to the attractive scalar mean field leads to an enhanced kaon yield in heavy-ion collisions at subthreshold energies. Also, kaon rescatterings are found to affect significantly the final kaon momentum spectra. We compare the theoretical results with recent experimental data from the Heavy-Ion Synchrotron (SIS) at GSI
\end{abstract}

PACS number(s): 25.75. $+\mathrm{r}, 21.65 .+\mathrm{f}, 24.10 . \mathrm{Cn}, 25.80 . \mathrm{Nv}$

Experiments on kaon production from heavy-ion collisions are being carried out at SIS [1]. The incident energy per nucleon in the collision is around $1 \mathrm{GeV}$ and is below the threshold energy of $1.56 \mathrm{GeV}$ for kaon production in the nucleon-nucleon collision in free space. One of the motivations for this study is to learn about the nuclear equation of state at high densities. As first pointed out in Ref. [2], using the Vlasov-Uehling-Uhlenbeck (VUU) model, kaon production from heavy-ion collisions at subthreshold energies offers the possibility of extracting the information about the nuclear equation of state as its yield can differ by a factor of three depending on the stiffness of the nuclear equation of state at high densities. A similar conclusion has been obtained recently using the covariant Boltzmann-Uehling-Uhlenbeck (BUU) model [3] and the quantum molecular dynamics $[4,5]$.

In the transport model for heavy-ion collisions, kaons are usually treated as free particles. In Ref. [6] the relativistic transport model has been generalized to include both the kaon mean-field potential and the collisions of kaons with other particles. In this Rapid Communication we shall use the generalized relativistic transport model to study subthreshold kaon production in heavy-ion collisions.

From the quantum hadrodynamics [7] in which the nuclear matter is treated as a system of interacting baryons and mesons, one can derive a relativistic transport equation for the phase space distribution function $f\left(x, \mathbf{p}^{*}\right)$ of nucleons [8]. This transport equation can be solved using the method of pseudoparticles in which each nucleon is replaced by a collection of test particles. The propagation of these test particles is described by the classical equations of motion,

$$
\begin{aligned}
& \frac{d \mathbf{x}}{d t}=\mathbf{p}^{*} / E^{*}, \\
& \frac{d \mathbf{p}}{d t}=-\nabla_{x}\left[E^{*}+\left(g_{\omega} / m_{\omega}\right)^{2} \rho_{N}\right],
\end{aligned}
$$

with $\rho_{N}$ being the nuclear matter density and $E^{*}=$ $\left(m^{* 2}+\mathbf{p}^{* 2}\right)^{1 / 2}$. The nucleon effective mass $m^{*}$ and ki-

\footnotetext{
*Permanent address: Institute of Atomic Energy, Beijing 102413, China.
}

netic momentum $\mathbf{p}^{*}$ are defined by

$$
m^{*}=m-g_{\sigma}\langle\sigma\rangle, \quad \mathbf{p}^{*}=\mathbf{p}-g_{\omega}\langle\omega\rangle .
$$

In the above, $g_{\sigma}$ and $g_{\omega}$ are the coupling constants of a nucleon to the scalar $(\sigma)$ and vector $(\omega)$ mesons, respectively. The expectation values of the scalar meson $\langle\sigma\rangle$ and the vector meson $\langle\omega\rangle$ are related in the mean-field approximation to the nuclear scalar and current densities.

The coupling constants are determined from the nuclear matter property. Two parameter sets have been obtained in Ref. [8] by choosing a nucleon effective mass $m^{*}=0.83 m$ at the normal nuclear density and allowing the scalar meson self-interactions. They correspond to a soft and a stiff nuclear equation of state with a compressibility of 200 and $380 \mathrm{MeV}$, respectively.

We also include deltas and treat their propagation to be similar to that of nucleons. Collisions among nucleons and deltas are treated as in the cascade model [9]. Besides elastic scatterings, both nucleons and deltas can interact inelastically via $N N \leftrightarrow N \Delta$. The pion degree of freedom is also included via $\Delta \leftrightarrow \pi N$, and it is assumed to propagate as a free particle in the nuclear medium.

Because of explicit chiral symmetry breaking, nucleons act on kaon as an effective scalar field $[10,11]$, i.e.,

$$
L_{s} \approx \frac{\Sigma_{K N}}{f_{K}^{2}} \bar{N} N \bar{K} K \text {. }
$$

This gives rise to an attractive $s$-wave interaction for the kaon. In the above, $f_{K}$ is the kaon decay constant and $\Sigma_{K N}$ is the $K N$ sigma term. Their values are taken to be $f_{K} \sim 93 \mathrm{MeV}$ and $\Sigma_{K N} \sim 350 \mathrm{MeV}$ as in Ref. [6]. There is also a vector interaction in the chiral Lagrangian,

$$
L_{v} \approx-\frac{3 i}{8 f_{K}^{2}} N^{\dagger} N \bar{K} \partial_{t} K,
$$

which leads to a repulsive $s$-wave interaction for a kaon in the nuclear matter. We note that the resulting vectorexchange mean-field potential between kaon and nucleon is just $1 / 3$ of the nucleon mean-field potential of Eq. (1) [12].

Combining the scalar and vector interactions with the 
kaon mass term, we obtain the kaon effective mass in the medium

$$
m_{K}^{* 2} \approx m_{K}^{2}\left(1-\frac{\Sigma_{K N} \rho_{s}}{f_{K}^{2} m_{K}^{2}}+\frac{3 \rho_{N}}{4 f_{K}^{2} m_{K}}\right),
$$

where $\rho_{s}$ is the nuclear scalar density. With $\Sigma_{K N} \sim 350$ $\mathrm{MeV}$, the kaon mass is seen to increase slightly with density. This is consistent with the empirical observation that the $K N s$-wave scattering length is negative but small. In Ref. [6], only the kaon self-energy due to the attractive scalar interaction is included in the kaon effective mass while that due to the repulsive vector interaction is included in the kaon energy as in the case of nucleons. In this case, the kaon mass in the medium is re- duced although its energy is increased. Since the kaon is a boson, the present approach of treating both the scalar and vector interactions on the same footing should be the consistent way for determining the kaon mass in a medium.

The dominant process for kaon production in heavyion collisions at subthreshold energies is from the baryonbaryon interaction $[13,14]$. The experimental cross section can be parametrized as [15]

$$
\sigma_{N N \rightarrow B Y K} \approx 72 p_{\max } / m_{K} \mu \mathrm{b}
$$

where $Y$ denotes $\Lambda$ or $\Sigma$ and $B$ denotes $N$ or $\Delta$. The maximum kaon momentum $p_{\max }$ is given by

$$
p_{\max }=\sqrt{\left[s-\left(m_{Y}+m_{B}+m_{K}\right)^{2}\right]\left[s-\left(m_{Y}+m_{B}-m_{K}\right)^{2}\right] / 4 s}
$$

Kaons can also be produced from nucleon-delta and delta-delta interactions. The corresponding cross sections have been estimated in Ref. [15] and it was found that $\bar{\sigma}_{N \Delta \rightarrow B Y K} \approx \frac{3}{4} \bar{\sigma}_{N N \rightarrow B Y K}$ and $\bar{\sigma}_{\Delta \Delta \rightarrow B Y K} \approx$ $\frac{1}{2} \bar{\sigma}_{N N \rightarrow B Y K}$ at the same center-of-mass energy.

To determine the kaon production cross section in the medium, we simply use the in-medium masses to evaluate $p_{\max }$. For the in-medium nucleon and kaon masses, we use those given by Eqs. (2) and (5). The hyperon mass in the medium can be obtained from the generalization of the Walecka model [16], i.e.,

$$
m_{Y}^{*}=m_{Y}-g_{\sigma Y Y}\langle\sigma\rangle
$$

where the hyperon-scalar meson coupling constant $g_{\sigma Y Y}$ is two-thirds of the nucleon-scalar meson coupling constant $g_{\sigma}$. Similarly, the vector mean-field potential for the hyperon is two-thirds of that for the nucleon.

The total center-of-mass energy $s^{1 / 2}$ of the colliding pair of baryons in Eq. (7) is given by

$$
s^{1 / 2}=\sqrt{m_{B_{1}}^{* 2}+\mathbf{p}^{* 2}}+\sqrt{m_{B_{2}}^{* 2}+\mathbf{p}^{* 2}}+\frac{1}{3}\left(\frac{g_{\omega}}{m_{\omega}}\right)^{2} \rho_{N}
$$

In the above, the last term accounts for the difference of the vector potential between the initial and final baryons. This additional energy is, however, cancelled by the vector potential of the kaon which has been included in the kaon effective mass. The vector potential energy is thus similar for the initial and final states so it does not play any role in particle production. As a result, the kaon production threshold is reduced by its attractive scalar interaction with nucleons.

Because of the small probability for kaon production in baryon-baryon interactions, kaon production in heavyion collisions at subthreshold energies is treated perturbatively so that the collision dynamics is not affected by the presence of produced kaons. When the energy in a baryon-baryon collision is above the threshold for kaon production, kaon is produced isotropically in the baryon-baryon center-of-mass frame with a momentum distribution taken to be the same as in free space [15], i.e.,

$$
\begin{aligned}
& \frac{E}{p^{2}} \frac{d^{2} \sigma_{B B \rightarrow B Y K}(\sqrt{s})}{d p d \Omega} \\
& =\sigma(\sqrt{s}) \frac{E}{4 \pi p^{2}} \frac{12}{p_{\max }}\left(1-\frac{p}{p_{\max }}\right)\left(\frac{p}{p_{\max }}\right)^{2}
\end{aligned}
$$

The invariant double differential cross section for kaon production in heavy-ion collisions is then obtained by summing over the impact parameter.

The propagation of kaons with in-medium mass is similar to that for nucleons. Representing kaons by test particles, their motions are given by equations similar to Eq. (1), i.e.,

$$
\begin{aligned}
\frac{d \mathbf{x}_{K}}{d t} & =\mathbf{p}_{K}^{*} / E_{K}^{*} \\
\frac{d \mathbf{p}_{K}}{d t} & =-\nabla_{x} E_{K}^{*}
\end{aligned}
$$

where the kaon energy is $E_{K}^{*}=\sqrt{m_{K}^{* 2}+\mathbf{p}_{K}^{* 2}}$. We note that the kaon vector potential has been included in its effective mass [see Eq. (5)].

To treat kaon rescatterings, we artificially allow $N_{K}$ kaons to be produced in each baryon-baryon collision with energy above the kaon production threshold [17]. Each kaon is then assigned a production probability, which is given by the ratio of the kaon production cross section to the baryon-baryon total cross section. The motions of kaons are then followed by solving the classical equations of motion given by Eq. (11).

The kaon-nucleon collision is included by using a kaonnucleon total cross section [18] of about $10 \mathrm{mb}$ which we take to be density independent. After the collision the kaon direction is isotropically distributed as the kaonnucleon interaction is mainly in the $s$ wave. Since kaon production is treated perturbatively, its effect on nucleon dynamics is neglected. We therefore do not allow the nucleon momentum to change in a kaon-nucleon interaction. 
We include also the collision between a kaon and a pion via the $K^{*}$ resonance [19]. Its effect in heavy-ion collisions at $1 \mathrm{GeV} /$ nucleon is, however, insignificant compared to the kaon-nucleon collision. This is due to both the smaller number of pions than nucleons in the system and the fact that pions materialize from delta decays in the expansion stage of heavy-ion collisions when the matter density starts to decrease.

To obtain the final kaon spectrum, we weight each kaon with the production probability introduced previously and then divide the sum by $N_{K}$. In the GSI experiment, only $K^{+}$is measured. To obtain theoretical $K^{+}$ yield, we need in principle to treat explicitly the isospin degree of freedom. For simplicity, we assume that the $K^{+}$yield is half of the kaon yield. This will be valid for heavy-ion collisions with isospin symmetric nuclei. For collisions involving isospin asymmetric nuclei, this assumption slightly overestimates the $K^{+}$yield.

To illustrate the above discussions and to understand the recent kaon data from the GSI, we have carried out a calculation for the collision of two Au nuclei at an incident energy of $1 \mathrm{GeV} /$ nucleon. Using the generalized relativistic transport model that includes the in-medium kaon mass and the kaon final-state interactions. The kaon momentum spectra obtained with a soft equation of state $(200 \mathrm{MeV})$ are shown in Fig. 1 for different laboratory angles. The experimental data at $44^{\circ}$ from SIS [1] are also shown in Fig. 1 by the open squares.

The magnitude of the calculated kaon yield is slightly below the measured one. We note, however, that we have not included in the calculation kaon production from the pion-nucleon interaction. This contribution has been shown to be about $25 \%$ of that from the baryon-baryon interaction [13]. The inclusion of kaon production from the pion-nucleon interaction is thus expected to bring our results closer to the experimental data. If the kaon self-energy due to the attractive scalar mean field is neglected in the calculation, the kaon yield is reduced by about a factor of 3 . This can be seen in Fig. 2 where

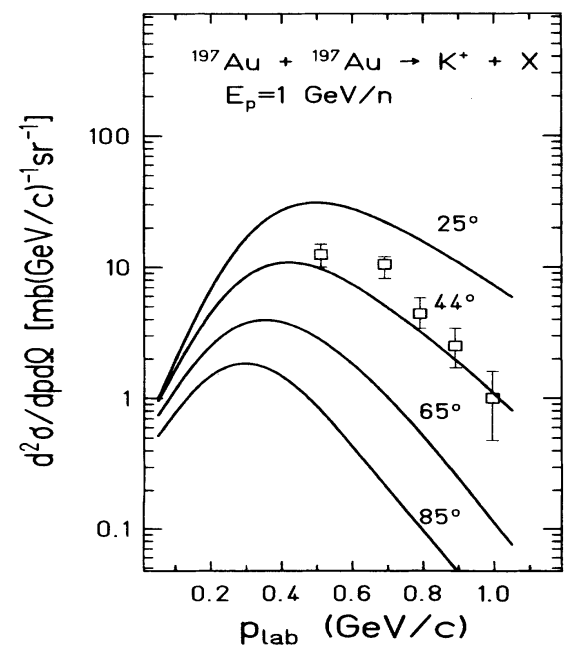

FIG. 1. Kaon momentum spectra at different laboratory angles for $\mathrm{Au}+\mathrm{Au}$ collisions at $1 \mathrm{GeV} /$ nucleon. The experimental data from Ref. [1] at $44^{\circ}$ are also shown.

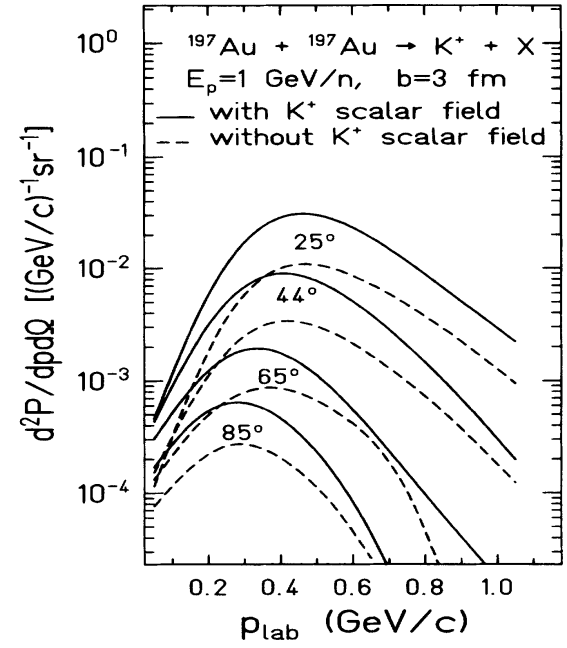

FIG. 2. Kaon momentum spectra for $\mathrm{Au}+\mathrm{Au}$ collisions at $1 \mathrm{GeV} /$ nucleon and at an impact parameter of $3 \mathrm{fm}$. Solid and dashed curves correspond to results with and without the kaon attractive scalar mean field, respectively.

the kaon momentum spectra for the same nuclear reaction at an impact parameter of $3 \mathrm{fm}$ are shown. Solid and dashed curves correspond to results obtained with and without the kaon attractive scalar mean field, respectively. In Refs. [4,5] the quantum molecular dynamics (QMD) with the Skyrme mean-field potential and the free kaon mass has been used to calculate the kaon yield, and their results agree also with the experimental data. However, it has been shown in Ref. $[5,20]$ that including the momentum-dependent interaction reduces the kaon yield by about a factor of 3 . Since our relativistic transport model includes implicitly the momentum-dependent effect, our results are thus consistent with those of Refs. $[4,5]$. Our calculation therefore indicates that the inclusion of kaon self-energy due to the attractive scalar meson field provides a plausible explanation of the measured kaon yield from GSI.

As can be seen in Fig. 1, the shape of the theoretical spectrum at $44^{\circ}$ is also consistent with the measured one. The kaon momentum and angular distribution are affected by its final-state interactions with nucleons. We find that on the average a kaon undergoes about five rescatterings as it is produced mainly in high density region. Neglecting the final-state interactions of kaons makes the kaon momentum spectra steeper and also reduces the kaon yield at large angles as in Refs. [17,21]. This effect is shown in Fig. 3 for the Au+Au reaction at an impact parameter of $3 \mathrm{fm}$. Solid curves are obtained with kaon rescatterings while dashed curves correspond to the case without such rescatterings.

All kaons are produced from the compression stage of the collision when the nuclear density is high. As in previous studies $[2-5,13]$, the delta-nucleon interaction accounts for more than half of the produced kaons.

The kaon yield does not change much if a stiff equation of state $(380 \mathrm{MeV})$ is used. This is different from the results obtained in the normal VUU/QMD model with 
the Skyrme mean-field potential where the dependence on the stiffness of the nuclear equation of state is more appreciable. This is due to the fact that in the relativistic transport model the sensitivity of the kaon yield to the nuclear compression energies is largely cancelled by the smaller effective nucleon mass in a soft equation of state than in a stiff equation of state at densities above the normal nuclear matter density. As pointed out in Ref. [2], the effect of nuclear equation of state on the kaon yield is more significant in heavy-ion collisions at lower incident energies such as $\sim 700 \mathrm{MeV}$. It is thus of interest to have experimental data on kaon yield at these energies.

In summary, we have generalized the relativistic transport model to include the kaon degree of freedom. Our results on the kaon yield for the collision between two $\mathrm{Au}$ nuclei agree with recent data from the SIS at GSI if the in-medium kaon mass is used. Neglecting its self-energy due to the attractive scalar mean field the kaon yield is about a factor of 3 smaller than the measured one. The final-state interactions of kaons with nucleons are found to increase appreciably the kaon yield at larger momenta and angles. However, we do not find any significant dependence of the kaon yield on the nuclear equation of state for heavy-ion collisions at $1 \mathrm{GeV} /$ nucleon. Due to the lack of data on kaon production from the nucleonnucleon interaction near the threshold and the complexity of the collision dynamics, our conclusion that the kaon has an appreciable attractive scalar mean-field potential in dense matter is thus tentative. With refined theoret-

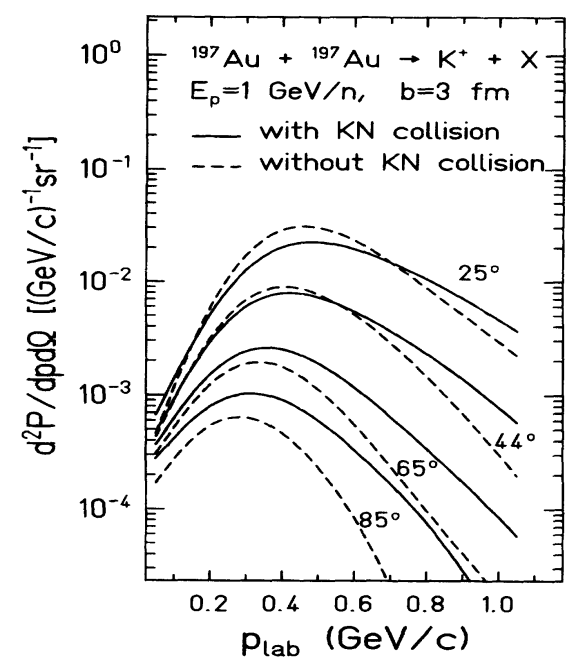

FIG. 3. Same as Fig. 2. Solid and dashed curves correspond to results with and without kaon final-state interactions, respectively.

ical studies and more experimental kaon data at larger angles and from lower incident energies, we shall be able to learn more about the property of kaon in dense matter and the nuclear equation of state at high densities.

This work was supported in part by the NSF Grant No. PHY-9212209 and the Welch Foundation Grant No. A-1110.
[1] E. Grosse, Prog. Part. Nucl. Phys. 30, 89 (1993).

[2] J. Aichelin and C. M. Ko, Phys. Rev. Lett. 55, 2661 (1985).

[3] A. Lange, W. Cassing, U. Mosel, and K. Weber, Nucl. Phys. A541, 507 (1992).

[4] G. Q. Li et al., Nucl. Phys. A537, 645 (1992); S. W. Huang et al., Phys. Lett. B 298, 41 (1993); G. Q. Li, A. Faessler, and S. W. Huang, Prog. Part. Nucl. Phys. 31, 159 (1993).

[5] G. Hartnack, J. Janicke, and J. Aichelin, Report No. LPN-93-11 (unpublished).

[6] X. S. Fang, C. M. Ko, and G. E. Brown, Phys. Rev. C 47, 1678 (1993).

[7] B. D. Serot and J. D. Walecka, in Advances in Nuclear Physics, edited by J. Negele and E. Vogt (Plenum, New York, 1986), Vol. 16

[8] C. M. Ko, Q. Li, and R. Wang, Phys. Rev. Lett. 59, 1084 (1987); Q. Li and C. M. Ko, Mod. Phys. Lett. A 3, 465 (1988); C. M. Ko and Q. Li, Phys. Rev. C 37, 2270 (1988); Q. Li, J. Q. Wu, and C. M. Ko, Phys. Rev. C 39, 84 (1989); C. M. Ko, Nucl. Phys. A495, 321c (1989).

[9] J. Cugnon, D. Kinet, and J. Vandermeullen, Nucl. Phys.
A379, 553 (1982).

[10] D. B. Kaplan and A. E. Nelson, Phys. Lett. B 175, 57 (1986); A. E. Nelson and D. B. Kaplan, Phys. Lett. B 192, 193 (1987).

[11] G. E. Brown, K. Kubodera, and M. Rho, Phys. Lett. B 193, 273 (1987).

[12] G. E. Brown, C. M. Ko, and K. Kubodera, Z. Phys. A 341, 301 (1992).

[13] L. Xiong, C. M. Ko, and J. Q. Wu, Phys. Rev. C 42, 2231 (1990).

[14] J. Q. Wu and C. M. Ko, Nucl. Phys. A499, 810 (1989).

[15] J. Randrup and C. M. Ko, Nucl. Phys. A343, 519 (1980); A411, 537 (1983).

[16] N. K. Glendenning and S. A. Moszkowski, Phys. Rev. Lett. 67, 2414 (1991).

[17] X. S. Fang, C. M. Ko, and Y. M. Zheng, Nucl. Phys. A556, 499 (1993).

[18] C. Dover and G. E. Walker, Phys. Rep. 89, 1 (1982).

[19] C. M. Ko, Phys. Rev. C 23, 2760 (1981).

[20] J. Aichelin, A. Rosenhauer, G. Peilert, H. Stöcker, and W. Greiner, Phys. Rev. Lett. 58, 1926 (1987).

[21] J. Randrup, Phys. Lett. 99B, 9 (1981). 